\title{
Condición periodontal en mujeres embarazadas entre los 20 y 35 años en el Hospital Metropolitano de Barranquilla
}

\author{
Edith de León; Leider Herazo; Alberto Hernández; Federman Zabala*; Guillermo Acosta Osio**; Jorge Coronell; \\ Campo Elías González***
}

\begin{abstract}
RESUMEN: Este es un estudio descriptivo hecho en 20 pacientes embarazadas seleccionadas al azar entre los 20 y 35 años de edad, que cursan diversos períodos del embarazo, atendidas en el Hospital Metropolitano de Barranquilla. El objetivo del estudio fue evaluar la presencia de placa y sangrado gingival en cada una de ellas mediante la exploración con sonda, según el método de Loe y Silness.

El análisis estadístico revela que la mayoría de pacientes tiene un índice de placa grado II, así como un índice gingival II. Se concluye que se debe concientizar a la mujer embarazada para que entienda que el embarazo no invariablemente se acompaña de enfermedad periodontal, pero que este período sí requiere una higiene oral más meticulosa. De la misma forma el obstetra debe recordar la necesidad del manejo interdisciplinario de la mujer embarazada como estrategia para mejorar la salud oral de sus pacientes.
\end{abstract}

PALABRAS CLAVES: Placa bacteriana, embarazadas, gingiva, manejo interdisciplinario.

SUMMARY: This is a descriptive investigation of 20 patients selected at random between 20 and 35 years old, in several phases of the pregnant attended at the Metropolitano's Hospital of Barranquilla. The objective was evaluated the presence of plate and gingival bleeding in each one of they, throug the exploration with probe, according to the loe and Silness'method.

The Statistic analysis reveals that the greater number of patients are found in a second degree of the plate index as well of the gingival index. It is concluded that it is neccessary to inform to the pregnant woman that the pegnancy not necessarily is accompanied of periodontal disease, but this period requires a more meticulous hygiene, is proposed as strategy that the physician recalls the need of interdisciplinary managing of the oral health in pregnant women.

KEY WORD: Plate, pregnant women, gingiva, interdisciplinary managing.

\section{Introducción}

Durante el período de gestación, la mujer se ve afectada en su cavidad oral por ciertos cambios en donde la encía varía de color normal a rojo intenso, brillante, lisa, con crecimiento focal frecuente e hiperemia intensa de la papila interdental, a estos cambios se le conoce como "Gingivitis del Embarazo" (1). Estos signos son desconocidos por la paciente, llevándola a deducir que son causa de su estado, despreocupándose por completo de su salud oral.

La gingivitis es un proceso inflamatorio de las encías que está asociado con hemorragias y exudación (3). La literatura científica se ha venido ocupando ampliamente del tema en cuestión, aceptando que el embarazo por sí mismo no causa gingivitis sino que es causada por irritantes locales como la placa bacteriana (2). La placa bacteriana se define como agregados microbianos ad-

Estudiantes de X Semestre de Odontología. U. Metropolitana. Director Departamento de Ginecología y Obstetricia, Universidad y Hospital Metropolitano.

Profesores Programa de Odontología. Universidad Metropolitana. heridos a los dientes u otras estructuras bucales sólidas, la cual desempeña un factor etiológico en el desarrollo de enfermedades periodontales, gingivales y caries dentales (8). La relación de la gingivitis y la cantidad de placa es igual después del parto que durante el embarazo.

Los cambios gingivales son indoloros a menos que se complique o se agreguen infecciones agudas o procesos pseudomembranosos (4). El mecanismo intrínseco de un desequilibrio hormonal no es descartado, ya que existen evidencias de que la progesterona afectare la función y permeabilidad gingivales (5). Sin embargo, la encía no vuelve a la normalidad después del parto mientras los irritantes locales estén presentes (6). Hay que descartar el papel de la microbiótica asociada a la gingivitis del embarazo, especialmente B. Melaninogenicus y Capnocytophaga, los cuales se hallan en grandes concentraciones, aceptándose como posible que contribuye a ello un elevado nivel hormonal, que actuaría como estimulante confluyendo a través de la saliva y el fluido crevicular (7).

Por último en este contexto puede quedar ubicado el presente estudio sobre gestantes, para evidenciar los porcentajes de placa y sangrado representativo de la 
condición periodontal de las mujeres embarazadas, y que además de preocuparse de su estado gestacional, se examine, trate y controle su salud oral.

\section{Material y métodos}

Fueron seleccionadas veinte pacientes al azar que cumplieron con las edades que oscilan entre 20 y 35 años, en varias fases del ciclo gestacional atendidas en el Hospital Metropolitano de Barranquilla.

El instrumental utilizado fue el siguiente: espejo plano, pinzas algodoneras, sonda periodontal tipo Marquis, sustancia reveladora de placa.

Para la determinación del índice de placa se emplea el método de Silnees y Loe (5) que mide la cantidad de placa depositada sobre la superficie de todos los dientes presentes en boca, según los siguientes criterios:

\section{Puntaje}

0

1

2

3

\section{Criterios}

No hay placa en zona gingival.

Una película de placa se adhiere al margen gingival libre y a la zona adyacente del diente.

2 Acumulo moderado de depósitos blandos sobre margen gingival, bolsa gingival o adyacente a la superficie del diente.

3 Abundancia de material blando dentro de la bolsa gingival o sobre el margen gingival y la superficie dentaria adyacente.

Según lo observado en la paciente, hemos dado un puntaje a cada una de las cuatro unidades gingivales del diente (vestibular, lingual, mesial y distal), siendo éste el índice de placa para la unidad gingival. Los puntajes de las cuatro caras del diente sumados y divididos por cuatro nos da el índice de placa del diente. Sumando los índices de la placa de cada diente y dividiéndolos por el número de piezas se obtiene el índice de placa de cada paciente.

Según el método de Silness y Loe, mide el estado de inflamación y sangrado que presenta la encía.

\section{Puntaje}

\section{Criterios}

$0 \quad$ Encía normal

1 Inflamación leve, ligero cambio de color, ligero edema, no sangra al sondar.

2 Inflamación moderada, enrojecimiento, edema, lisura, sangra al sondar.

3 Inflamación marcada, enrojecimiento, edema, ulceración y tendencia a la hemorragia espontánea.

La evaluación de los índices gingivales es semejante, por superficie y piezas presentes en boca, que el índice de placa.

Los datos obtenidos se consignaron en un formato que consta del nombre de la paciente, edad, mes de gestación, fecha. Seguidamente se encuentra un recuadro en el que aparece la nomenclatura de los dientes y caras de éstos, cada diente presenta 2 columnas en la cual la del lado derecho corresponde a la presencia de placa y el lado izquierdo al sangrado y luego siguen los resultados del índice de placa y del índice gingival de la paciente y por último las observaciones.

\section{Resultados y comentarios}

El número total de mujeres embarazadas observadas fue de 20 pacientes. Examinando las gráficas podemos observar que en el índice de placa la mayor frecuencia de pacientes están entre los índices de placa 1,18 y 1,42 (Gráfica 1). Y la mayor cantidad de pacientes se encuentra por encima de uno (1), lo cual indica que el $90 \%$ prevaleció en el Criterio 2 (Gráfica 3).

En el índice gingival la mayor frecuencia de pacientes se encuentra entre los índices gingival 0.94 y 1.13 (Gráfica 2) y el mayor número de pacientes se encuentra por encima de uno (1), lo cual indica que el $80 \%$ de pacientes se encuentran en el Criterio 2 (Gráfica 4).

Los resultados obtenidos están de acuerdo con el trabajo realizado por Gerardo Rodríguez (5), donde encontró que la respuesta gingival está aumentada durante la gestación a los irritantes locales, a pesar que en el trabajo realizado por este autor, que examinó a 32 mujeres gestantes, de diferentes edades y 10 pacientes no gestantes, las cuales fueron sometidas a un tratamiento a base de higiene profesional, educación sanitaria y aplicación tópica de flúor; mientras que en nuestra investigación el estudio se realizó en 20 pacientes gestantes con

\section{Gráfica 1}

\section{DIAGRAMA INDICE DE PLACA}

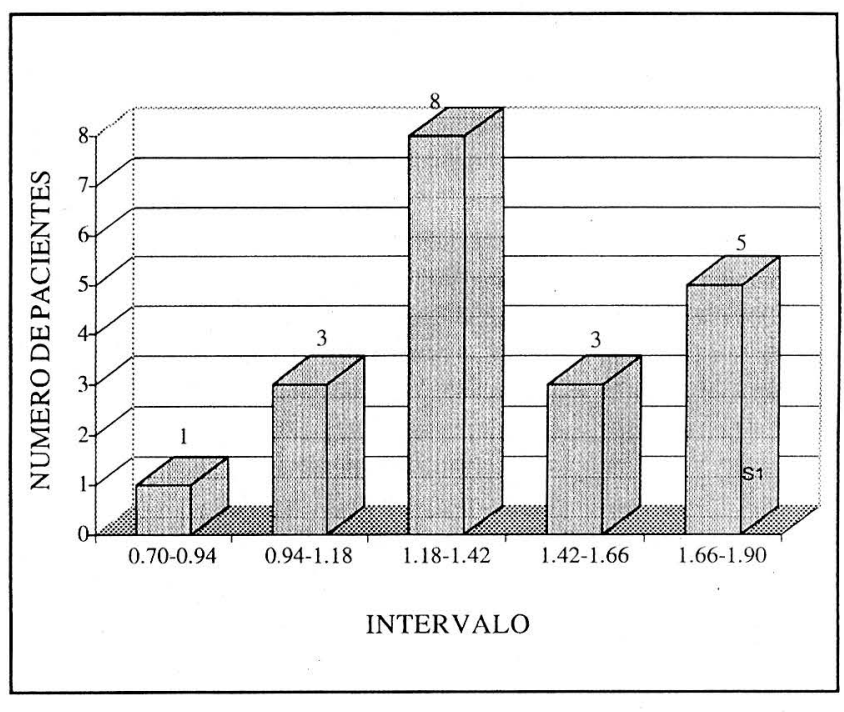

Grado 1: 0.7-0.94

Grado 2: $0.94-1.90$

Grado 3: $1.90-2.40$

La mayor frecuencia de pacientes (8) se encuentra entre los intervalos $1.18-1.42$

El mayor número de pacientes se encuentra en el Grado 2. 
Gráfica 2

DIAGRAMA INDICE GINGIVAL

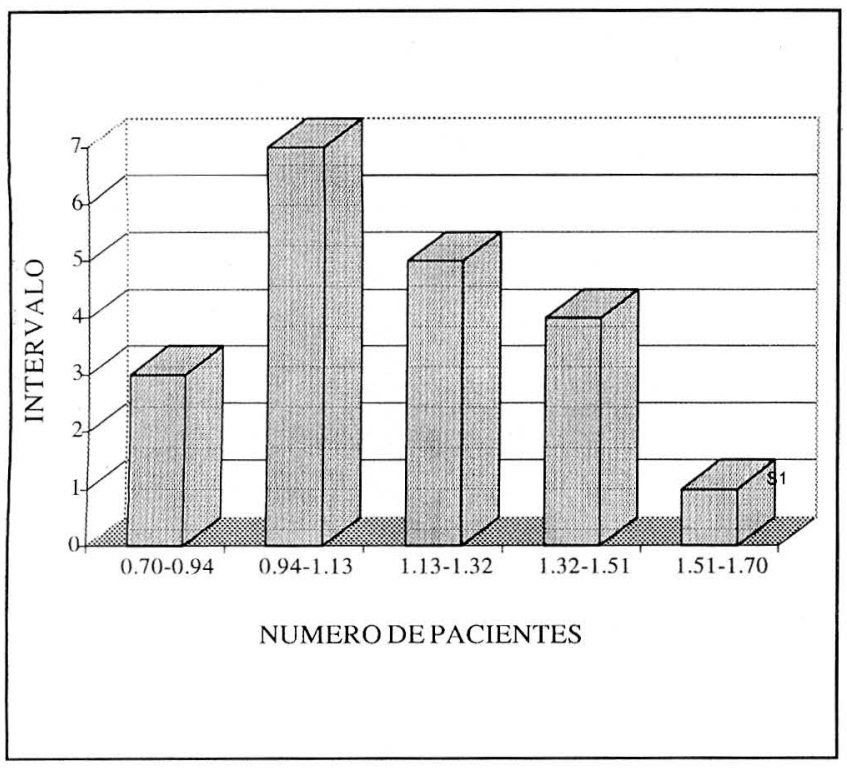

Grado 1: 0.7-0.94

Grado 2: $0.94-1.80$

Grado 3: $1.80-2.80$

La mayor frecuencia de pacientes (7) se encuentra entre el intervalo $0.94-1.13$

El mayor número de pacientes (16) se encuentra en el Grado 2.

Gráfica 3

DIAGRAMA DE PLACA (\%)

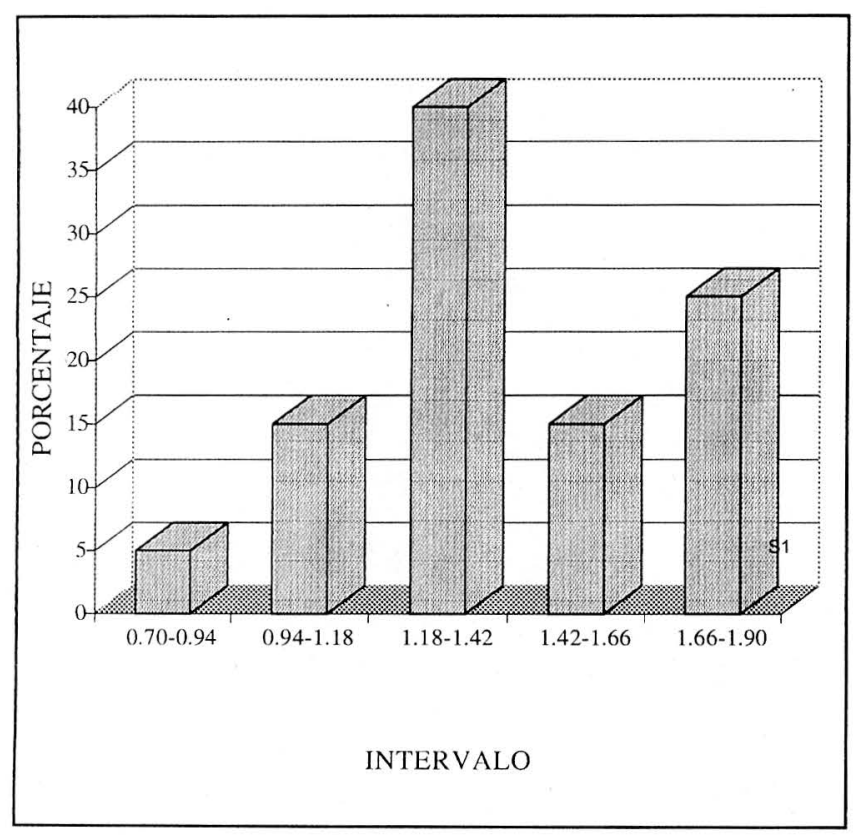

Grado 1: 0.70-0.94

Grado 2: 0.94-1.90

Grado 3: $1.90-2.40$
Gráfica 4

DIAGRAMA INDICE GINGIVAL (\%)

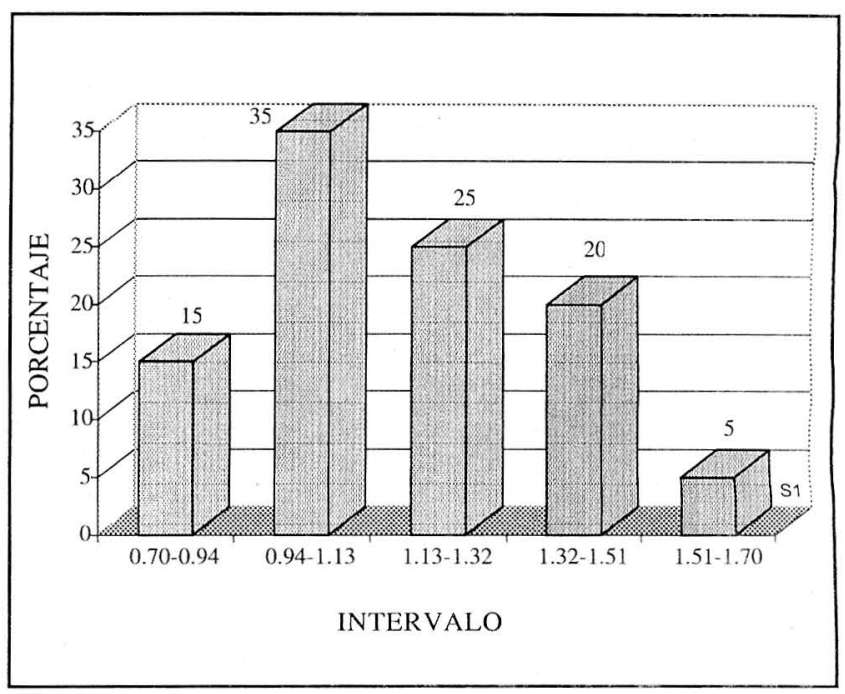

Grado 1: $0.75-0.94$

Grado 2: $0.94-1.80$

Grado 3: $1.80-2.80$

edades entre 20 a 35 años y no fueron sometidas a ningún tratamiento ni evaluación ya que este trabajo se enfocó en la evaluación de la condición periodontal (Indice de Placa- Indice Gingival), se han presentado similitud en estas dos investigaciones en los factores que influyen a la formación de placa como el flujo salival, la mala higiene oral, los cuales modifican la respuesta normal de la encía.

También nuestros resultados están de acuerdo con los trabajos de Maier y Orban citados por Rodríguez (5) en donde la respuesta gingival durante la gestación está acentuada a los irritantes locales o algún condicionamiento adicional a la placa. Se diferencian de nuestro trabajo en que ellos examinan a no embarazadas, y nosotros encontramos mayor volumen de gingivitis en pacientes embarazadas $(5)$.

\section{Conclusiones y recomendaciones}

De acuerdo con los resultados obtenidos notamos que la placa bacteriana está presente en un alto porcentaje en las gestantes entre los 20 y 35 años atendidas en el Departamento de Ginecología y Obstetricia del Hospital Universitario Metropolitano, como consecuencia de esto el índice gingival también se ve aumentado por la presencia de irritantes locales, lo que nos indica que la higiene oral en estas pacientes es bastante deficiente. Por consiguiente; la respuesta gingival está incrementada por la presencia de la placa bacteriana, lo cual modifica el cuadro clínico de estas pacientes.

Ante esto, podemos afirmar que este irritante es el principal agente etiológico de los diferentes cambios presentados en la encía en el período de gestación, entre los cuales encontramos sangrado al sondaje y al cepillado, enrojecimiento de la encía, textura lisa, aumento de tamaño y consistencia blanda. 
Conociendo lo anterior, recomendamos concientizar a la mujer embarazada que el embarazo no es un estado que necesariamente tenga que afectar la cavidad oral, sino un período en el cual la higiene oral debe ser más meticulosa.

De igual manera, sugerimos vincular dentro de la historia clínica de ginecobstetricia un anexo que permita evaluar los síntomas presentes en la cavidad oral de la paciente, para que el ginecólogo la remita al odontólogo y que éste la evalúe estomatológicamente, le transmita las instrucciones necesarias y realice el tratamiento respectivo. De esta manera, se establece interdisciplinariedad entre la Ginecobstetricia y la Odontología, con el fin de brindar una mejor atención de forma integral a la mujer embarazada que acude al Hospital Universitario Metropolitano en la consulta prenatal.

Recordamos que es de gran importancia el control odontológico durante el embarazo, ya que mediante este se le llevará a cabo tratamiento de periodoncia, en el cual se eliminarán los irritantes locales como medida profiláctica de salud oral e incluso general y de esta forma, se disminuyen a un mínimo las molestias a nivel de la cavidad oral.

\section{BIBLIOGRAFIA}

1. Undhe, Jan. Gingivitis del Embarazo Periodontología Clínica. Editorial Médica Panamericana. Buenos Aires, 1986; 250.

2. Shaiffer, W.G. Gingivitis de Embarazo. Tratado de Patología Bucal. 4a. edición. Editorial Interamericana. México, 1987; 806.

3. Jabloski. Placa Dental y Gingivitis. Diccionario Ilustrado de Odontología, Editorial Panamericana. Buenos Aires, 1992; 810-915.

4. Carranza, F.H. Gingivitis del Embarazo. Periodontología Clínica de Glickman. 7a. edición. Editorial Panamericana. México, 1970; 188491.
5. Rodríguez Baceiro, Gerardo. Influencia de la Higiene oral en la Gingivitis del Embarazo. Revista de Actualidad Estomatológica Española. Revista. Vol. 47 (367) Madrid. 1987; 29-30.

6. Hugonson. Gingival Inflamations and Tamales Sex Hormonas J. Periodontal Res. 5 (suppl), 1970.

7. Korman y Luesche. The Subgingival Microbial Flora During Pregnancy. J. Periodont. Res. 1980; 15: 111.

8. Daweg y cols. The Effect of Hormonal J. Periodontal. Res. 1974; 9: 18. 